\title{
Reply to CME
}

\section{Continuous medical education activities; Answers to Case No. 1}

\author{
Said Hamed Abbadi* \\ Medical Microbiology and Immunology, Faculty of Medicine, Suiz University
}

\author{
A R T I C L E I N F O \\ Article history: \\ Received 1 April 2020 \\ Received in revised form 4 April 2020 \\ Accepted 4 April 2020
}

\section{Keywords:}

Respiratory infection

Children

Fever

\begin{abstract}
During the winter months (Late November to early April), respiratory illnesses caused by both respiratory syncytial virus (RSV) and influenza virus are frequently epidemic in children 5 years of age and younger. Because the clinical presentation of these 2 illnesses in children can be similar, it is useful to distinguish between the two in the seriously ill child. The rapid enzyme immunoassay test which can detect antigens of either influenza A or B directly in respiratory secretions was positive for influenza A virus. In addition to influenza A virus (the most common and most likely to cause severe disease), infection can occur with influenza B virus (also common) and with influenza $\mathrm{C}$ virus (less common, less sever, and does not occur in epidemics). Influenza viruses are single-stranded, segmented, negative sense RNA viruses.

Influenza viruses have two well characterized virulence factors on the surface of the virus, neuraminidase and hemagglutinin. Neuraminidase mediate virus penetration through the mucus layer overlaying the surface of the respiratory epithelium, and it also plays a role in the release and spread of the virus from infected respiratory cells.

Once the virus penetrates to the surface of the cells, binding to specific sialic acid reach receptors is mediated by hemagglutinin. Also, hemagglutinin plays a role in the formation of channels through which viral RNA can enter the cytoplasm and initiate the viral replicative cycle.
\end{abstract}

1. During the winter months (Late November to early April), respiratory illnesses caused by both respiratory syncytial virus (RSV) and influenza virus are frequently epidemic in children 5 years of age and younger. Because the clinical presentation of these 2 illnesses in children can be similar, it is useful to distinguish between the two in the seriously ill child.

2. The rapid enzyme immunoassay test which can detect antigens of either influenza A or B directly in respiratory secretions was positive for influenza $\mathrm{A}$ virus. In addition to influenza $A$ virus (the most common and most likely to cause severe disease), infection can occur with influenza $\mathrm{B}$ virus (also common) and with influenza $\mathrm{C}$ virus (less common, less sever, and does not occur in epidemics). Influenza viruses are single-stranded, segmented, negative sense RNA viruses. Influenza viruses have two well characterized virulence factors on the surface of the virus, neuraminidase and hemagglutinin. Neuraminidase mediate virus penetration through the mucus layer overlaying the surface of the respiratory epithelium, and it also plays a role in the release and spread of the virus from infected respiratory cells. Once the virus penetrates to the surface of the cells, binding to specific sialic acid reach receptors is mediated by hemagglutinin. Also, hemagglutinin plays 
a role in the formation of channels through which viral RNA can enter the cytoplasm and initiate the viral replicative cycle.

3. Most cases of influenza in this age group are selflimiting. However, several observations have been made concerning influenza in no immunocompromised children. During flu season, the number of hospitalizations for respiratory illnesses, outpatient visits, cases of severe bacterial pneumonia and the use of antibiotics all increase. Influenza is a much greater threat to individuals over 50 years of age. Especially those with underlying chronic diseases, particularly cardiopulmonary cases. Other patient populations recognized to be at increased risk for adverse outcomes with influenza infection include children with asthma and other chronic lung diseases, residents of long-term care facilities and women in the second or third trimester of pregnancy.

4. The physician was concerned that the patient might have Kawasaki disease, a generalized vasculitis seen most frequently in children less than 5 years of age. This patient has many of the signs associated with this syndrome, including prolonged fever, bilateral conjunctivitis, cracked and bleeding lips, swollen extremities and lymphadenopathy.

5. Reye's syndrome occurs primarily in children who are treated with aspirin (salicylates) during viral infection, particularly that caused by influenza viruses or varicella virus (chicken pox). This syndrome is characterized by fatty degeneration of the liver as well as acute encephalopathy with cerebral edema.

6. Day care centres have been shown to be an environment in which influenza A viruses can spread rapidly. Since many of his classmates were sick during a time of the year (January) when both influenza A and RSV are likely to be endemic in the community, it would not be surprising that he became infected in his day care centre. In addition, the observation that his one-year old brother also was sick with a syndrome consistent with influenza points out that a child in a day care can bring home a variety of infectious agents including influenza, resulting in illness among family members.

7. Influenza can be prevented by the use of a trivalent inactivated vaccine. The strains present in the current vaccine include two subtypes of influenza $\mathrm{A}, \mathrm{H} 1 \mathrm{~N} 1$ and
H3N2, and influenza B. This vaccine must be given annually, as immunity to the virus is short lived. The antigenic composition of the vaccine is determined by the types of viruses that circulated during the previous season. The efficacy of the vaccine is dependent on the level of change that may occur from year to year in the circulating virus. Changing in the antigenic structure of influenza A are classified in two ways, as 'antigenic drift' and 'antigenic shift'. The antigenic variations occur because of changes in the hemagglutinin $(\mathrm{H})$ glycoprotein antigen and the neuraminidase $(\mathrm{N})$ glycoprotein antigen. A total of three subtypes of hemagglutinin ( $\mathrm{H} 1, \mathrm{H} 2$, and $\mathrm{H} 3)$ and two subtypes of neuraminidase ( $\mathrm{N} 1$ and $\mathrm{N} 2$ ) are typically associated with human influenza A virus epidemics.

The vaccine is given to at risk populations and health care providers who could transmit the virus to their atrisk patients. Recent studies suggest that immunocompetent children may also benefit from vaccination through reduction in hospitalizations, doctor office visits, antibiotic use, serious secondary bacterial infections, and spread to at risk family members. The vaccine is not recommended for children less than 6 months of age. A live, attenuated influenza vaccine that can be given intranasally has been shown to be protective in children older than 15 months.

8. There are currently two classes of anti-influenza drugs. The first classes of agents block formation of influenza-derived ion channels. The reason these virally derived ion channels are important is that they play an important role in the uncoating of the virus. This is a step in viral replication in which viral nucleic acid, in this case RNA, is released from the viral particle and enters the cytoplasm of the cell. The two drugs in this class are the oral agents amantadine and rimantadine.

The second group of agents is the neuraminidase inhibitors. Two agents belong to this class of drugs; zanamivir, which is inhaled agent, and oseltamivir, which is an oral agent.

\section{References}

1- Abbadi SH. Continuous medical education activities, Case No. 1. Microbes Infect Dis 2020; 1 (1): 4-5. 